\title{
The MSDP of THEMIS: Capabilities, first results and prospects
}

\author{
P. Mein ${ }^{\star}$
}

Observatoire de Paris, Section de Meudon, 92195 Meudon Cedex, France

Received 17 April 2001 / Accepted 14 June 2001

\begin{abstract}
We present some capabilities and results of the Multi Channel Subtractive Double Pass instrument of THEMIS. More details and references can be found at http://www.themis.iac.es. MSDP imaging spectropolarimetry is characterized by high space and time resolution. The spatial resolution is close to seeing limitations, and large areas of the Sun can be scanned within short time intervals. In the case of THEMIS, polarization measurements are possible. 2 Stokes parameters and 16 wavelengths of a line profile can be observed simultaneously in a 2D field of view. The scattered light is reduced by the use of the long predisperser as a first-pass spectrograph. Some results are presented for fine structures of the chromosphere and stratification of magnetic fields. The MSDP of THEMIS is very efficient to observe fast extended events, such as eruptive phenomena and mass ejections, in particular during coordinated campaigns with space instruments. General outlines of the dataprocessing are given. All codes are available at http://BASS2000.bagn.obs-mip.fr. In conclusion, we mention future prospects with respect to spatial and spectral resolution, large field-of-view, multi-line observations, and compatibility with other THEMIS observing modes.
\end{abstract}

Key words. techniques: spectroscopy - techniques: polarimetry - Sun: atmosphere - Sun: magnetic fields

\section{Introduction}

Solar research includes a wide range of topics, and high performances are required by scientific programs.

THEMIS is designed to run several complementary exchangeable observing modes. It is unique in this respect.

- The slit-spectroscopy (MTR) observes many lines in 1D field-of-view with high spectral resolution,

- The narrow-band filter (IPM) provides 2D-fields in a number of wavelengths successively, with high spatial resolution,

- The MSDP records simultaneously a number of wavelengths in 2D fields, with high temporal and spatial resolution.

We do not approach here the general description of the telescope, designed by Jean Rayrole, neither the MTR and IPM modes. We concentrate on the MSDP mode and its capabilities for imaging spectro-polarimetry.

Additional information can be found at http://www.themis.iac.es.

\footnotetext{
* e-mail: Pierre.Mein@obspm.fr
}

\section{MSDP imaging spectroscopy: High resolution in space and time}

Since 1970, several MSDPs were built and operated in several telescopes:

- the Meudon Solar Tower (Mein 1977)

- the Turret Dome of Pic-du-Midi (Mein 1981)

- the German VTT telescope of Tenerife (Mein 1991)

- the Wroclaw large coronagraph (Rompolt B. 1993) The THEMIS MSDP is the most powerful one. We shall detail in the next section its specific capabilities. Let us recall that, as did the previous ones, this MSDP extracts a 2D-field of the solar image (typically $10^{\prime \prime} \times 170^{\prime \prime}$ ) and dispatches the photons over a number of $2 \mathrm{D}$ channels (typically 16 for one line profile) recorded by CCD cameras. The pixel size is around 0.2 arcsec.

In order to achieve the best spatial resolution in imaging spectroscopy, the MSDP avoids the convolution by a slit-width (unlike the slit-spectroscopy) and differential blurring between successive wavelengths by seeing effects (unlike narrow-band filters). A general description of the MSDP principle can be found in Mein (1995).

By using successive steps across the solar disk, large areas can be scanned within short times. The speed of the MSDPs is appreciated in coordinated campaigns involving ground-based and space telescopes. 


\section{Specific capabilities of the THEMIS-MSDP}

The following scheme shows the successive parts of the MSDP layout:

Polarization-free telescope

$$
\Downarrow
$$

First focus F1:

Grid for slice selection

Polarizer and beamsplitter

(see 3.6)

$\Downarrow$

Telescope transfer optics

$\Downarrow$

Second focus F2:

Field-stop

$\Downarrow$

Long predisperser

(see 3.1)

$\Downarrow$

Lenses for dispersion adjustment

Intermediate focus:

MSDP multi-slits and beamshifters

(see 3.2, 3.3, 3.4, 3.5)

$\Downarrow$

Echelle spectrograph

$\Downarrow$

Detector transfer optics

CCD cameras

(see 3.7)

The MSDP is characterized by a set of equipment and capabilities which cannot be found in other instruments. We review them briefly.

\subsection{Long "predisperser"}

Previous MSDPs used two passes on a single grating to produce images (subtractive double pass). THEMIS takes advantage of a long predisperser for the first pass, while the main spectrograph is only used for the second pass. Among the 3 exchangeable gratings of the predisperser, the MSDP uses the echelle grating similar to the grating of the main spectrograph. A two-lens optics adjusts the focal lengths, so as to get a perfect subtraction of the dispersions between the first and second spectrographs. Using two gratings instead of one significantly reduces the scattered light. Interferential filters before the SP1 optics (typical bandwidth $100 \AA$ ) are used to sort the orders of the two echelle gratings.

\subsection{New beamshifters}

The key parts of the MSDP are the "beamshifters" made of multiple slits and prisms. They select the wavelengths and translate the beams in the intermediate focus SP1 (between both passes) to create the channels. The focal lengths of the spectrographs, roughly $8 \mathrm{~m}$, are smaller than

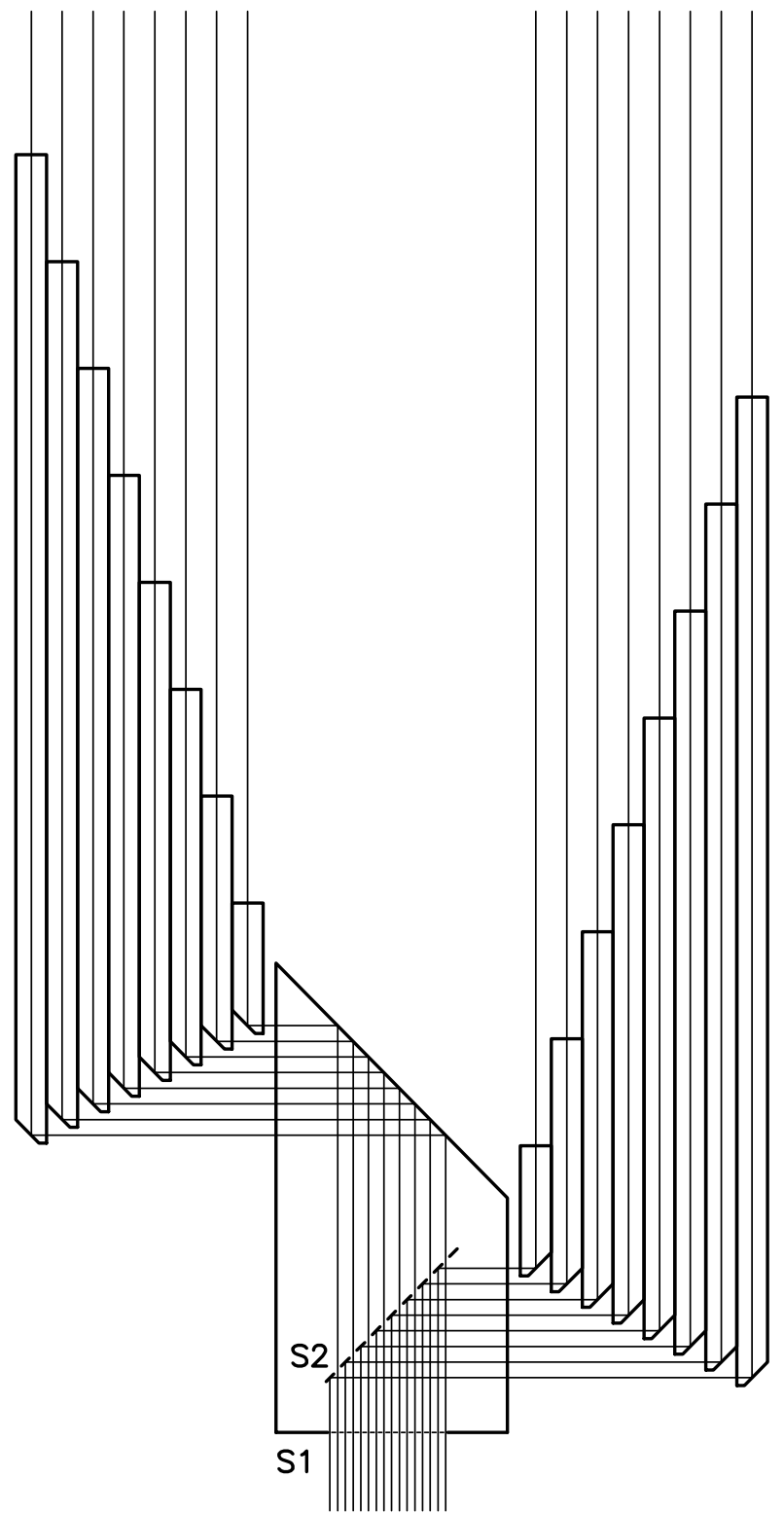

Fig. 1. Principle of the new MSDP beamshifter for high spectral resolution. The multiple slit S1 selects the wavelengths. Odd beams are reflected by the second multiple slit $\mathrm{S} 2$.

the lengths of the MSDP in Meudon (14 m) or in the VTT of Tenerife $(15 \mathrm{~m})$. In order to keep comparable spectral resolutions, we developped a new kind of "beamshifter". Figure 1 shows the principle of the 16 channel optics of THEMIS (B, C in Table 1). The wavelengths are selected by a first multiple slit $\mathrm{S} 1$. The second multiple slit S2 reflects the odd beams, while the even beams go through. Two series of 8 prisms compensate for the differences in optical paths.

The beamshifter of optics A (Table 1) are generally used for broad Balmer lines, which do not require high spectral resolution. It is one of the beamshifters built for the MSDP of the German VTT (Mein 1991, Fig. 4a). 


\subsection{Adjustable spectral resolution for high spatial resolution}

The step of the multiple slit S1 defines the spectral resolution. A larger step means a larger width of the slits, shorter integration times and accordingly higher spatial resolutions of the spectro-images. The mechanical mounts of all the MSDP beamshifters are similar, so that the optics of all MSDPs are exchangeable. Table 1 presents the specifications of some available beamshifters (optics D should be in operation in 2002). Spectral resolution and bandwidths of the slits are given around $600 \mathrm{~nm}$. Examples of corresponding lines of interest are also mentioned.

\subsection{Scanning step and spectral coverage}

Several successive exposures can be used to observe large areas of the solar disk. The scanning step cannot exceed the width of the field-stop allowed by the beamshifter. Another constraint follows from the desired spectral coverage, that is, the wavelength interval available for each line profile. If $N$ is the number of channels, $\delta \lambda$ the spectral resolution (distance between channels), and $\partial \lambda / \partial x$ the dispersion of the spectrograph, the spectral coverage $\Delta \lambda$ can be deduced from the scan-step $\Delta x$ by the relationship (Mein 1991)

$\Delta \lambda=(N-1) \delta \lambda-\Delta x \partial \lambda / \partial x$.

Table 1 presents the field-stop sizes as well as the usual values of scanning steps for different optics and lines. Let us take an example. At $600 \mathrm{~nm}$, the dispersion is roughly $6 \mathrm{pm}$ per arcsec. With the optics $\mathrm{B}$ or $\mathrm{C},(N-1) \delta \lambda$ is $15 \times 8 \mathrm{pm}=120 \mathrm{pm}$, and a $6^{\prime \prime}$ scan-step corresponds to the wavelength interval $120-6 \times 6=84 \mathrm{pm}$.

The scan-step determines also the number of exposures necessary to observe the intensity line profile (Stokes $I$ ) in a given field of view (Table 1, Col. 8).

\subsection{Multi-line observations}

Two lines can be observed simultaneously with two of the optics mentioned in Table 1. However, the two necessary megapixel cameras will be available only during the observing campaigns of 2001. Successive observations of different lines can also be performed by fast automatic rotation of the gratings. We shall come back in Sect. 6 to the possible extension to 3 or 4 simultaneous lines.

\subsection{Polarimetry}

THEMIS is a $90 \mathrm{~cm}$ polarization-free telescope, especially designed for polarimetric observations. For the first time, MSDP observations of a magnetic field can be performed extensively. The polarization analyser designed by J. Rayrole provides successively the Stokes parameters $I \pm S(S=V, Q$, or $U)$ without differential blurring due to seeing effects. It is located at the primary focus F1. Because the accuracy of measurements is very sensitive to small differences between the optical paths corresponding to both states of polarization $I \pm S$, the MSDP is generally used with a "grid" (Semel 1980) located before the analyser. The period of the grid is twice the distance between the two images produced by the beam splitter (typically $34^{\prime \prime}$ ). At the entrance of the spectrographs (focus F2), the splitting is oriented in the direction perpendicular to the dispersion. Figure 2 shows an example of raw data obtained with the circular polarization analysis and the optics C.

At a given time, the grid selects about $17^{\prime \prime}$ of solar disk at each period of $34^{\prime \prime}$. Three successive exposures are necessary to reconstruct the full image with sufficient overlaps. In Table 1, Col. 9 shows the number of necessary exposures for $I \pm V$ observations of a $120^{\prime \prime} \times 170^{\prime \prime}$ field of view. For full $I, Q, U, V$ observations, 3 times more exposures are necessary (last column).

It can be noted that, if some error occurs in the rotation of the image due to the transfer optics between F1 and F2, the 2D character of the MSDP allows correction off-line of the orientation of the translation between polarized beams $I \pm S$ created by the beamsplitter and the grid. Let us remark that, since the distance between both images is close to 17 arcsec, a 1-degree error leads only to a shift of 0.3 arcsec.

\subsection{High speed detectors}

New fast CCD cameras (Berrilli et al. 1999) will be installed during the next observing campaign. We may expect time intervals smaller than $2 \mathrm{~s}$ between successive exposures. It follows from Table 1 that the observation of $I \pm V$ across a $120^{\prime \prime} \times 170^{\prime \prime}$ field-of-view should last less than 2 min with optics B or C, and less than 1 min with optics A. In case of small solar features and $24^{\prime \prime} \times 170^{\prime \prime}$ field-of-view, less than $24 \mathrm{~s}$ and $12 \mathrm{~s}$ should be sufficient respectively.

\section{Data processing}

The data are available one year after the date of the observations at http://BASS2000.bagn.obs-mip.fr. The software is also available, with the user's guide, and some sets of parameters for typical observations.

The data processing consists of several steps detailed below.

\subsection{Geometrical calibrations}

This step determines the accurate correspondence between pixels of all channels across the solar image. The geometry is specified by the edges of channels (maximum gradient of the intensity signal). For this purpose, observations are performed in the continuum close to the line, to get similar intensities in all channels.

Control plots are produced at each step of the processing. Residual distortions can be taken into account. Plots 
Table 1. Optics A, B, C, D: number of channels, approximate spectral bandwidths and resolutions (distances between channels) at $600 \mathrm{~nm}$, relevant lines, associated field-stops and usual scanning steps, corresponding numbers of exposures for a $120^{\prime \prime} \times 170^{\prime \prime}$ field of view.

\begin{tabular}{|c|c|c|c|c|c|c|c|c|c|}
\hline Optics & $\begin{array}{r}\text { Number of } \\
\text { channels }\end{array}$ & $\begin{array}{r}\text { bandwidth } \\
(\mathrm{pm})\end{array}$ & $\begin{array}{r}\text { resol. } \\
(\mathrm{pm})\end{array}$ & $\begin{array}{l}\text { lines } \\
(\mathrm{nm})\end{array}$ & field-stop & $\begin{array}{l}\text { scan } \\
\text { step }\end{array}$ & \multicolumn{3}{|c|}{$\begin{array}{l}\text { number of exposures } \\
\text { for } 120^{\prime \prime} \times 170^{\prime \prime}\end{array}$} \\
\hline A & 9 & 8 & 24 & $486.1,656.3$ & $25^{\prime \prime} \times 170^{\prime \prime}$ & $12^{\prime \prime}$ & 10 & 30 & 90 \\
\hline $\mathrm{B}, \mathrm{C}$ & 16 & 4 & 8 & $\begin{array}{l}517.3,518.4 \\
587.6,589.0 \\
589.6,854.2\end{array}$ & $9^{\prime \prime} \times 170^{\prime \prime}$ & $6^{\prime \prime}$ & 20 & 60 & 180 \\
\hline $\mathrm{D}$ & 14 & 2 & 5 & 610.3 & $9^{\prime \prime} \times 170^{\prime \prime}$ & $4^{\prime \prime}$ & 30 & 90 & 270 \\
\hline
\end{tabular}

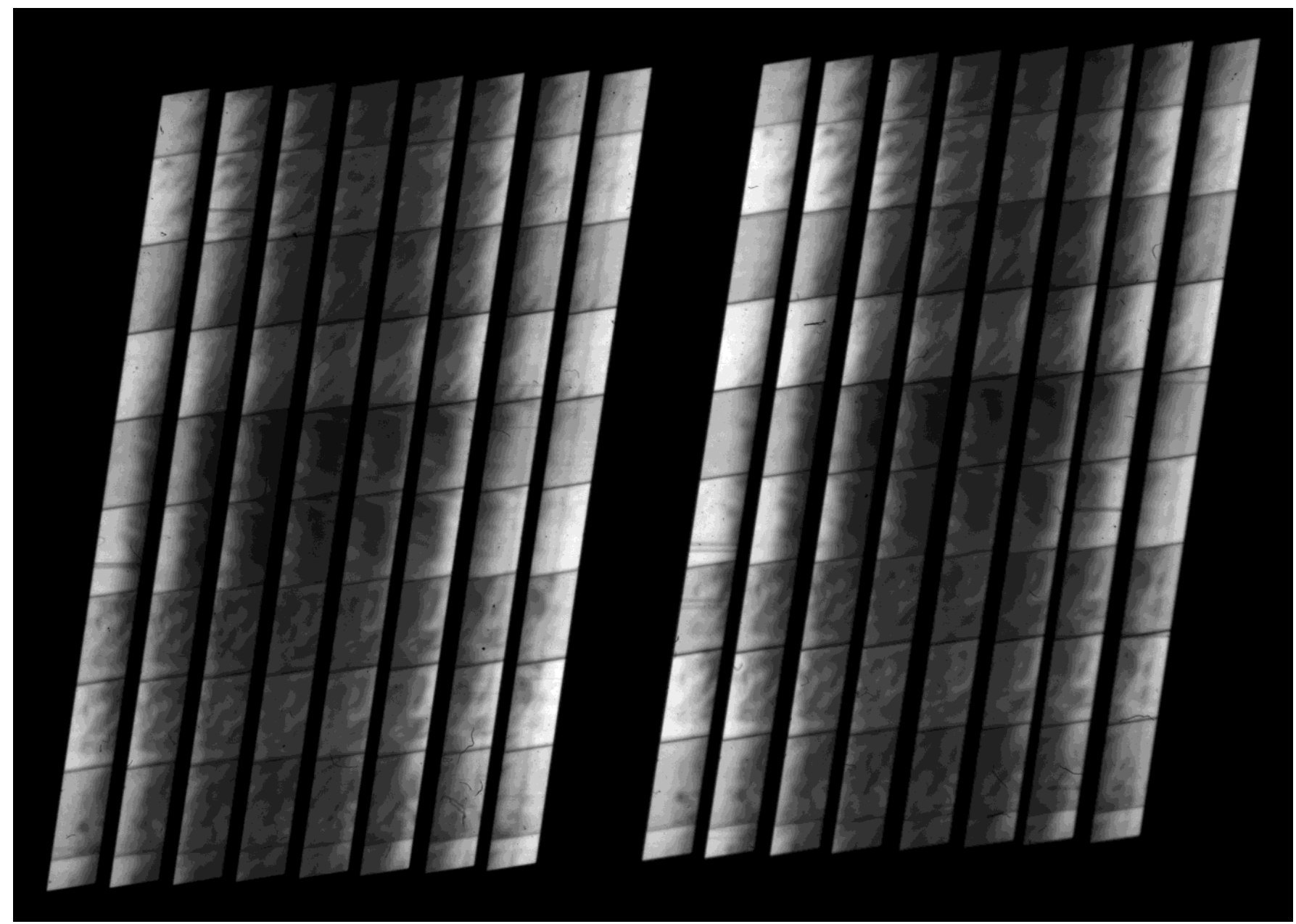

Fig. 2. $\mathrm{H}_{\alpha}$ MSDP image obtained with a grid in front of the polarization analyser. The same fields of view are seen simultaneously in $I+V$ and $I-V$, so that the Stokes parameters are not affected by differential seeing effects. Similar images can be obtained for $Q$ or $U$. The wavelengths of lefthand channels are intercalated in between the wavelengths of righthand channels. (Courtesy C. Fang).

of the geometrical components of the rectangular field versus the channel-number are used to check the accuracy. The maximum departure between results and regression lines is generally smaller than 0.2 arcsec.

\subsection{Photometric calibrations}

Photometric calibrations are deduced from dark current records and from flat field observations in the line profile.
Pointing at the line profile instead of the continuum is necessary to remove fringes and instrumental effects. If small shifts occur with respect to geometrical calibration images (because of grating rotations), they are corrected by global translations.

In all channels, the constant-wavelength curves are approximated by second degree curves (curvature of spectral lines). A mean profile of the spectral line is obtained by 
averaging the signal along these curves. Wavelength overlaps of successive channels are used to determine the relative transmissions. For each channel, a 2D array is produced to specify the photometric calibration.

\subsection{Wavelength calibration}

The wavelength defining the zero Doppler velocity can be deduced from the line center of flat-field profiles. However, the solar rotation must be taken into account. Generally the line-center wavelength is derived from a preliminary dopplergram of the full observed field-of view (quick-look).

\subsection{Dopplergrams, magnetograms and line profiles}

Scans are performed across the disk, with steps between $4^{\prime \prime}$ and $12^{\prime \prime}$, according to the line. Two kinds of maps can be produced:

- Intensity maps (spectroheliograms) at a set of wavelengths, derived from spline interpolations between the channels. Various algorithms are available to improve the accuracy of spline interpolations. Line profiles (and Stokes profiles) can be restored from these maps, at any point in the field-of-view.

- Dopplergrams (and magnetograms of $B_{\|}$) are derived from the bisector method, at different levels in the line profile.

\section{Examples of results}

Although the focal instrumentation is still under development (see Sect. 6), the performances already meet the requirements of many scientific programs. We present some examples.

\subsection{Fast chromospheric dopplergrams}

1) During a ground-space campaign with $\mathrm{SOHO}$, filaments were observed in $\mathrm{H}_{\alpha}$ on May 14, 2000 with optics A. We present in Fig. 3 a line-center image and a dopplergram at $\pm 24 \mathrm{pm}$. Detailed analysis, including power spectra of oscillations, are performed in comparison with CDS results (Régnier et al. 2001).

2) Penumbral waves and umbral flashes have been observed on Aug. 21, 2000 with optics C in CaII $8542 \AA$. Using a $23 \mathrm{mn}$ time series, Tziotziou et al. (2002) investigate their spatial and temporal behaviour. The MSDP is well adapted to fast chromospheric events.

\subsection{Stratification of magnetic fields}

On May 9, 2000, an active region was observed in the NaD1 line, with polarization analysis and optics C. Figure 4 shows the line-of-sight magnetic field derived from the bisector method at $\pm 16 \mathrm{pm}$. Figure 5 presents some $I$ and $V$ profiles along the line ab crossing two spots of opposite polarities. Magnetograms have been computed
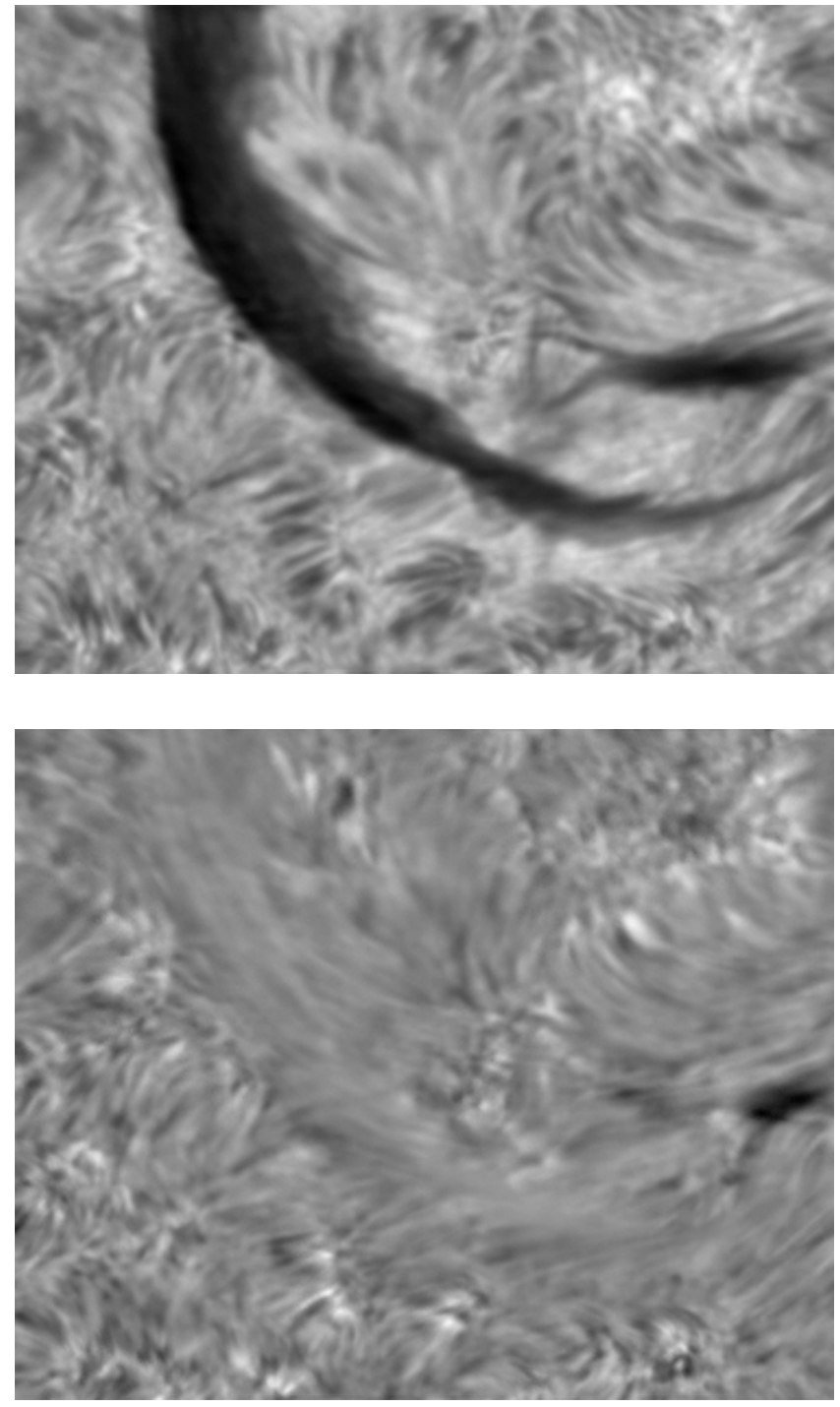

Fig. 3. $H_{\alpha}$ filament observed on 14 May 2000. Line center intensity (top) and Doppler velocity (bottom) computed at $\pm 24 \mathrm{pm}$ with the bisector method. The field of view is $150^{\prime \prime} \times 123^{\prime \prime}$ (Courtesy S. Régnier).

also for $\pm 8 \mathrm{pm}$ and $\pm 24 \mathrm{pm}$. They provide the magnetic field at different levels of the photosphere and the low chromosphere. By using NLTE response functions, Eibe et al. (2002) compared the vertical gradients with the gradients deduced from force-free extrapolations of the field into the corona.

\subsection{Ground-space observations}

In addition to the observations mentioned in Sect. 5.1, several campaigns were coordinated with SOHO, TRACE and YOHKOH.

1) Arch Filament Systems were observed on Sep. 10, 1998 and analysed in terms of a cloud model (Mein P. et al. 2000). TRACE, VTT-MSDP and THEMIS-MSDP data were used to determine temperatures and densities (Mein N. et al. 2001). 


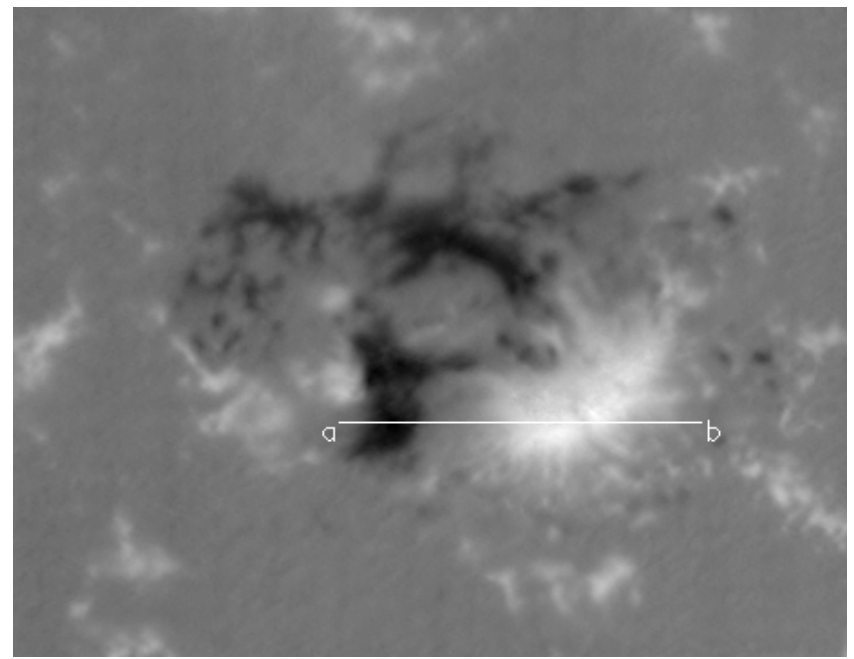

Fig. 4. Longitudinal magnetic field deduced from MSDP observations in the NaD1 profile on May 9, 2000. Bisector method, at $\pm 16 \mathrm{pm}$. Polarization analysis with grid in the primary focus (Sect. 3.6). The field of view is $138^{\prime \prime} \times 120^{\prime \prime}$. (Courtesy J.-M. Malherbe).

2) A coordinated campaign with SOHO/CDS and SUMER was devoted to filament absorptions on May 5, 2000 (Schmieder B. et al. 2001). Results were obtained about the opacity of Lyman continuum compared to $\mathrm{H}_{\alpha}$.

\section{Prospects}

Specific scientific programs should require increased performance. We mention some improvements concerning either new equipment or new observing procedures and processing.

\subsection{High spatial resolution: Destretching and speckle imaging}

There are at least two ways to increase the spatial resolution.

The first one is adaptive optics. This should be installed at THEMIS during the next few years. It should provide very high resolution inside the isoplanetism domain, that is, less than one minute of arc (with corrections in one pupil only).

The second one consists of off-line corrections by destretching and/or speckle restoration, when short exposure times are available to freeze the telluric turbulence. We plan to take advantage of new fast CCDs to record bursts of images. By adding the signals in all the MSDP channels, it is possible to build low-noise images (equivalent to the white channel in the case of narrow-band filters). They can be used to determine the destretching parameters.

Such processing can improve the spatial resolution in large fields of view. It can be noted that, in the case of polarization analysis, the size of each instantaneous

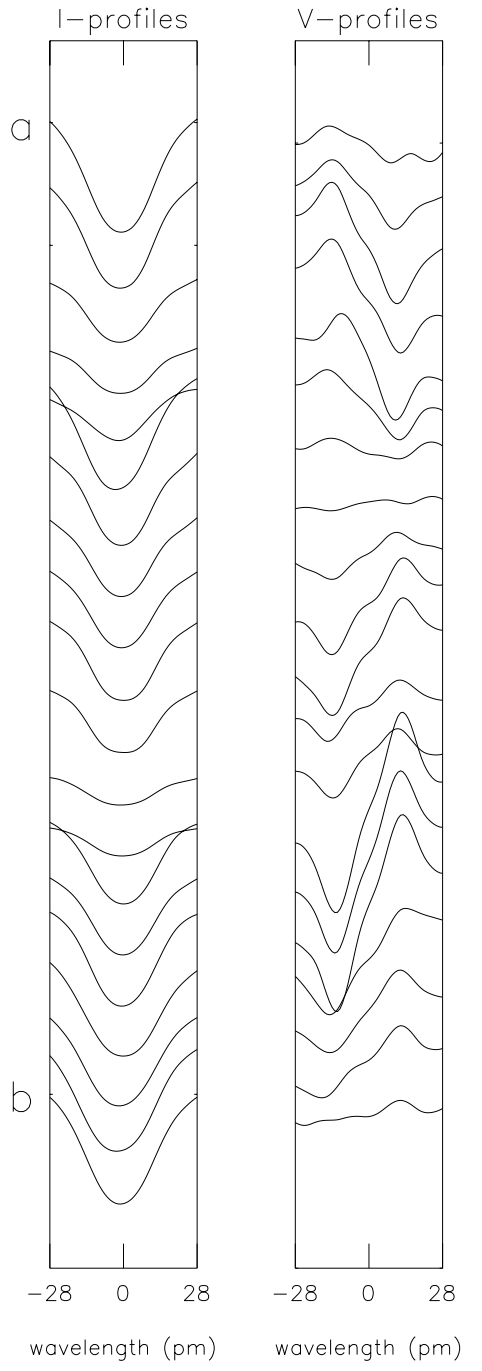

Fig. 5. $I$ - and $V$-profiles of NaD1 along the line ab of Fig. 4. Two spots of opposite polarities are crossed. (Courtesy J.-M. Malherbe).

image created by the grid is close to isoplanetism domains (around $10^{\prime \prime} \times 10^{\prime \prime}$ ). This can be useful for destretching procedures.

The best equipment for high spatial resolution should consist of large fast cameras (at least $1500 \times$ 1000 pixels, 2 to $5 \mathrm{~Hz}$ ) and/or optical enlargement at the F2 focus (typically $\times 1.5$ ). In addition, a project using a very bright beamshifter is ready (18 channels, widthslits $12 \mathrm{pm}$, resolution $12 \mathrm{pm}$ ). It should allow very short exposure times, freezing the telluric turbulence $(\sim 10 \mathrm{~ms})$ for strong lines.

High resolution is crucial in spectro-polarimetry of flux tubes, fine structure of flares, or threads of filaments acting as magnetic tracers of the low corona.

\subsection{High speed capabilities}

Table 1 shows that the expected speeds in 2001 allow the instrument to scan a $120^{\prime \prime} \times 170^{\prime \prime}$ field of view within $120 \mathrm{~s}$ or less, in 2 Stokes parameters (optics B or C). With larger pixel sizes, for example 0.4 arcsec, the same time should 
apply to $120^{\prime \prime} \times 240^{\prime \prime}\left(240^{\prime \prime}\right.$ is the maximum simultaneous field of THEMIS).

Fast evolutions of magnetic and velocity fields can be recorded over full active regions. Many programs are relevant: wave propagation, flares and mass ejections, photosphere-corona investigations from coordinated ground-space observations with large fields-of-view.

\subsection{Large and fast scans using earth rotation}

It is quite possible to scan the solar disk with the motion of earth rotation. Scan-steps of 7.5 arcsec imply only a rate of 2 exposures per second. Successful trials have been performed during 1999 with the small THEMIS CCDs, without polarization analysis. The exposure times were around $50 \mathrm{~ms}$. For polarization analysis and larger exposure times, it would be necessary to compensate for the earth's velocity during the integration time. This might be done, either by the telescope transfer optics, or by a rotating plate at the $\mathrm{F} 2$ focus. It can be noted that the use of earth rotation allows any scanning length, while the scanning by the telescope transfer optics alone cannot exceed 4 min of arc.

As an example of a relevant program, we can mention proper motions of magnetic tracers, which require a very uniform scanning velocity.

\subsection{Improved spectral resolution}

It could be possible to increase the spectral resolution and the number of channels by a factor of 2 with beamsplitters slightly inclined in front of the optics A, B, C or D. In Table 1, the spectral resolutions would become respectively 12,4 and $2.5 \mathrm{pm}$. The field-of-view would be reduced by a factor of 2 , but the number of channels would be increased by the same factor (respectively 18, 32, 32 and 28).

This would be especially interesting for investigations of photospheric lines with optics B, C and D.

\subsection{Multi-line imaging-spectropolarimetry}

Multi-line observations are a specific advantage of spectrographs, unlike narrow-band filters. The plane mirrors feeding the MSDP beamshifters allow the simultaneous observation of 2 lines. To exchange lines, the adjustments are still difficult. New devices could reduce the times, and extend the capabilities to 3 or 4 lines.

All investigations concerning the stratification of the solar atmosphere should take advantage of such improvements.

\subsection{Improved polarimetric accuracy}

The accuracy of polarization measurements depends on the possible integration over time and space. The 2Dcharacter of the MSDP can be used to increase the integration by $2 \mathrm{D}$-smoothing. This does not degrade the spatial resolution, because exposure times are generally long for weak polarization observations, except in the case of very efficient adaptive optics. The smoothing leading to the best compromise can be optimized off-line with MSDP data.

\subsection{Compatibilities MSDP/MTR and MSDP/IPM}

The output corresponding to optics A and B coincides with the standard focus of the MTR mode. If the focus F2 was equipped with an automatic exchange of slits and field-stops, fast successive observations could be possible with MTR and MSDP. It must be noted also that the CCD used for the MSDP can be useful in performing slit-spectroscopy in Additive Double Pass, with the two echelle gratings. The dispersion is almost twice the usual MTR dispersion. The spectral range remains large because of the large CCD.

The MSDP and IPM modes are already compatible, in the case of observations without polarization analysis. Polarimetry should be operational in both cases in the future. Two new CCDs are required; MSDP and IPM cannot use the same detectors at the same time.

\section{Conclusion}

The highest MSDP performances are available at THEMIS. In addition to high spatial and temporal resolutions, polarimetric analysis is available in 2D spectroscopy. Very suitable for observations of fine magnetic features, the MSDP can also efficiently follow fast evolutions of extended unstable structures, such as eruptive events and mass ejections. In ground-space coordinated campaigns, the 2D character of MSDP allows easy superposition on data from other instruments.

New improvements can be expected in the future with respect to spatial, spectral and temporal resolutions, as well as polarimetric accuracy and number of lines observed simultaneously.

With the complementary capabilities of the three observing modes, MTR, IPM and MSDP, THEMIS should meet the requirements of most of the new problems of solar physics.

Acknowledgements. We would like to thank J. Rayrole who included the MSDP in the general layout of THEMIS, the members of CNRS-INSU who made possible the construction of the instrument, and the THEMIS team who operates the telescope at Tenerife. We would like to thank also the SERT of the Paris Observatory, C. Coutard, R. Hellier, F. Colson and the engineers of the solar department who made many parts and adjustments for the MSDP, C. Briand who efficiently performed all the MSDP observations, and the solar data base BASS2000 that dispatches the data to observers from Tarbes and Meudon. THEMIS is a French-Italian telescope operated on the island of Tenerife by CNRS-CNR in the Spanish Observatorio del Teide of the Instituto de Astrofisica de Canarias. 


\section{References}

Berrilli, F., Cantarano, S., Egidi, A., et al. 1999, JOSO Ann. Rep. 1999, 29, 92

Eibe, M. T., Aulanier, G., Faurobert, M., Mein, P., \& Malherbe, J.-M. 2002, A\&A, 381, 290

Mein, N., Schmieder, B., DeLuca, E. E., et al. 2001, ApJ, 556, 438

Mein, P. 1981, Proceedings of the Japan-France seminar on solar physics, ed. F. Moriyama, \& J.-C. Hénoux, 285

Mein, P. 1991, A\&A, 248, 669

Mein, P. 1995, 3D Optical Spectroscopic Methods in Astronomy, ed. G. Comte, \& M. Marcelin, ASP Conf. Ser., 71,350
Mein, P., Briand, C., Heinzel, P., \& Mein, N. 2000, A\&A, 355, 1146

Régnier, S., Solomon, J., Mein, P., Briand, C., \& Vial, J.-C. 2001, A\&A, in preparation

Rompolt, B., Mein, P., Mein, N., Rudawy, P., \& Berlicki, A. 1993, JOSO Ann. Rep., 87

Schmieder, B., Tziotziou, K., Heinzel, P., \& Malherbe, J.-M. 2001, Themis and the new frontiers of solar atmosphere dynamics, FSAD2001 meeting, Rome, submitted

Semel, M. 1980, A\&A, 91, 369

Tziotziou, K., Tsiropoula, G., \& Mein, P. 2002, A\&A, 381, 279 